\title{
Eugenol como anestésico para oscar, Astronotus ocellatus
}

\author{
Silva-Souza, J.G. ${ }^{\circledR}$; Andrade, D.R. ${ }^{1}$; Vidal Júnior, M.V. ${ }^{1}$; Farias, W.M. ${ }^{2}$; Valério Júnior, J.F. ${ }^{2}$ e Mendonça, P.P. ${ }^{2}$ \\ 'Universidade Estadual Norte Fluminense Darcy Ribeiro. RJ. Brasil. \\ ${ }^{2}$ Instituto Federal de Educação, Ciência e Tecnologia do Espírito Santo. Rodovia. Brasil.
}

\section{PalaVRas chaVe adicionals}

Estágios de sedação.

Estágios de recuperação.

Peixe ornamental.

Óleo de cravo.

\section{RESUMO}

A utilização de anestésicos durante práticas de manejo na piscicultura é considerada uma medida mitigadora do estresse dos peixes. A espécie utilizada no experimento, o oscar, é um peixe nativo da Bacia Amazônica de grande importância para a piscicultura ornamental e amplamente difundida na aquariofilia mundial. O objetivo deste trabalho foi avaliar o efeito anestésico do eugenol sobre juvenis de oscar. Foram utilizados juvenis com peso de $4,61 \pm 0,83 \mathrm{~g}$ e comprimento total de 6,36 $\pm 0,32 \mathrm{~cm}$ submetidos a diferentes concentrações de eugenol na água, em aquários distintos e posteriormente foi observada sua recuperação em aquários sem eugenol. Foram avaliadas quatro concentrações de eugenol: 40; 60; 80 e $100 \mathrm{mg} / \mathrm{L}$. Os valores referentes ao tempo de permanência nos estágios de sedação e recuperação, bem como o tempo para completa sedação e retorno à natação normal foram avaliados e analisados via ANOVA $(p<0,05)$ e regressão polinomial. Foi observado que quanto maior a concentração, menor foi o tempo de indução à anestesia dos peixes. Para a recuperação não ocorreu efeito entre as concentrações de 60,80 e 100 mg/L. A melhor concentração de eugenol para a sedação dos juvenis de oscar é $80 \mathrm{mg} / \mathrm{L}$.

\section{Eugenol as anesthetic for oscar, Astronotus ocellatus}

\section{SUMMARY}

The use of anesthetics in fish farming is a practice to control fish stress. The oscar is a native fish from Amazon and it is of great importance to ornamental fish farming around the World. The aim of this experiment was to evaluate the sedative effect of eugenol in juvenile oscar, it used juveniles with body weight of $4.61 \pm 0.83 \mathrm{~g}$ and total length of $6.36 \pm 0.32 \mathrm{~cm}$. These juveniles were exposed to different concentrations of eugenol and then placed for recovery in aquariums with no eugenol. Four doses were evaluated: 40;60;80 and 100 $\mathrm{mg} / \mathrm{L}$. The time values of sedation stages and recovery stages, as well as the amount of time to complete sedation and return to normal swimming were analyzed using ANOVA $(p<0.05)$ and polynomial regression. The author observed that the higher the concentration of eugenol is, the less the sedation time is. In the recovery stage, there was no significant effect to the concentrations of 60,80 and $100 \mathrm{mg} / \mathrm{L}$. The best concentration of eugenol to sedation of juvenile oscar was $80 \mathrm{mg} / \mathrm{L}$.

\section{INFORMACIÓN}

Cronología del artículo.

Recibido/Received: 11.12.2014

Aceptado/Accepted: 22.6.2015

On-line: 16.9.2015

Correspondencia a los autores/Contact e-mail:

igilmar.souza@gmail.com

\section{INTRODUÇÃO}

O comércio de peixes ornamentais é uma atividade que movimenta grande montante financeiro no Brasil e no mundo (Magalhães and Jacobi, 2013). Características particulares do processo de produção de peixes ornamentais fazem com que esta seja considerada uma atividade agropecuária rentável (Zuanon et al., 2006). Uma das grandes vantagens da piscicultura ornamental é o seu baixo custo de produção, o que permite a implantação em pequenas áreas e utilização de mãode-obra familiar (Cardoso et al., 2012).
O oscar (Astronotus ocellatus), também conhecido como apaiari ou acará-açu, é um ciclídeo ornamental carnívoro, nativo da Bacia Amazônica (Trindade and Queiroz, 2012). Esta espécie é conhecida pela tolerância a baixas taxas de oxigênio (Wood et al., 2009), pelo temperamento calmo e comportamento territorialista, e possui importância econômica no Brasil e no mundo. Está entre as espécies nativas de valor para a aquicultura ornamental (Ribeiro et al., 2007).

$\mathrm{Na}$ piscicultura os peixes estão sujeitos a muitos agentes estressores que, em situações extremas, po- 
Tabela I. Estágios de sedação e recuperação observados utilizando eugenol como anestésico para juvenis de Astronotus ocelattus (Sedation and recovery stages observed for juveniles Astronotus ocelattus anesthetized with eugenol).

\begin{tabular}{ll}
\hline ESTÁGıO & DESCRIçÃo Do ESTÁGIO \\
\hline Normal & Reaçães e natação normal \\
Sedação leve & Perda de reação a movimentos visuais e ao toque. \\
Anestesia leve & Perda parcial de equilíbrio. \\
Anestesia profunda & Perda total de equilíbrio. \\
Anestesia cirúrgica & Movimento mínimo dos opérculos, e peixes estáticos. \\
Regularização dos batimentos operculares & Retomada de ritmo constante nos batimentos operculares. \\
Início de movimentação & Retomada de movimentos corporais. \\
Natação errática & Natação irregular sem direção controlada com o corpo fora do equilíbrio normal. \\
Recuperação final & Natação regular, com o corpo em equilíbrio, porém sem reação à estímulos externos. \\
Recuperado & Natação e reação normais. \\
\hline
\end{tabular}

dem afetar a saúde e o bem-estar dos animais; o uso de anestésicos é uma alternativa para a redução dos efeitos nocivos do estresse sobre os animais (Ashley, 2006). A anestesia pode auxiliar a diminuição do estresse reduzindo a atividade do sistema nervoso central reduzindo a atividade locomotora. Manejos que exijam exposição ao ar como biometrias, deslocamento dos peixes para outras estruturas de criação ou aquários, e avaliação do estado sanitário dos peixes seriam facilitadas com a utilização de anestésicos.

Anestésicos sintéticos já são amplamente utilizados para estes fins, a exemplo da benzocaína, 2-phenoxietanol e a tricaína metano-sulfonato (Inoue et al., 2004; Holloway et al., 2004). Atualmente, a utilização de anestésicos de origem natural é cada vez mais frequente na piscicultura (Façanha et al., 2005; Becker et al., 2012; Benovit et al., 2012). Existe uma vasta gama de substâncias naturais com potencial utilização na aquicultura, o óleo-de-cravo (eugenol) é um composto natural com ampla utilização em peixes e de fácil aquisição.

$\mathrm{O}$ eugenol $\left(\mathrm{C}_{10} \mathrm{H}_{12} \mathrm{O}_{2}\right)$ é um óleo essencial extraído das gemas florais do cravo-da-índia que possui múltiplos usos, sendo utilizado principalmente como antisséptico e anestésico local (Costa, 2000). Existem experiências positivas de sua utilização como anestésico para muitas espécies de peixes, como o lambari, esturjão, tilápia e a piraputanga (Pereira-da-Silva et al., 2009; Imapoor et al., 2010; Delbon and Ranzani Paiva, 2012; Fabiani et al., 2013). O eugenol apresenta baixo risco ao manipulador e aos peixes, sendo rapidamente metabolizado e eliminado dos tecidos e da corrente sanguínea (Hoseini et al., 2015). Por ser um composto de baixa solubilidade em água, para utilizar o eugenol é comum que seja feita a diluição em etanol, as proporções de diluição variam, é comum encontrar na literatura recomendações de 1:20 ou de 1:10 na proporção de eugenol:etanol (Inoue et al., 2011; Souza et al., 2012). Para determinação das concentrações adequadas para anestesia de peixes é necessário estudar os seus efeitos sobre o comportamento dos animais.

A anestesia ocorre em diferentes estágios que vão desde a diminuição das respostas aos estímulos externos até a completa perda de equilíbrio, movimentos corporais e respiratórios (Parodi et al., 2012). O estado normal de atividade é o ponto de partida para as avaliações do comportamento dos peixes ao ter contato com o anestésico. É importante conhecer aspectos com- portamentais da espécie em estudo, principalmente atividade natatória e reação a estímulos como o toque. Brycons e salmonídeos são grupos de peixes que apresentam maior atividade natatória e agressividade (Arbaláez-Rojas and Moraes, 2009) e, portanto, terão reação comportamental diferente daquela apresentada por peixes com menor atividade. A alteração do comportamento normal pode ser constatada pela redução na atividade natatória e fim de hiperatividade.

O primeiro sinal de ação do anestésico é a perda de reação a estímulos externos, como o toque e estímulos visuais, e redução nos batimentos operculares, neste primeiro momento não ocorre perda de equilíbrio (Gimbo et al., 2008; Teixeira et al., 2011). Persistida a sedação, ocorrem alterações no equilíbrio natatório do peixe (Simões et al., 2010). Inicialmente a natação é comprometida de forma a se tornar irregular e desorientada até cessar e o peixe perde totalmente o equilíbrio. Após a perda de equilíbrio o peixe perde os movimentos, os batimentos operculares se tornam mínimos e, caso a sedação seja continuada, o peixe pode morrer (Anschau et al., 2014) . Da mesma forma que a sedação, a recuperação também ocorre em estágios sequenciais, iniciando com a regularização dos batimentos operculares, posteriormente iniciam-se os movimentos corporais, início de recuperação da natação e recuperação de natação normal (Zahl and Samuelsen, 2012). O objetivo deste trabalho foi avaliar o efeito anestésico do eugenol, bem como recuperação anestésica em diferentes concentrações sobre juvenis de oscar.

\section{MATERIAL E MÉTODOS}

O experimento foi realizado no mês de setembro de 2014, na área de experimentação do Laboratório de Nutrição e Produção de Espécies Ornamentais (LNPEO) do Instituto Federal de Educação, Ciência e Tecnologia do Espírito Santo, Campus de Alegre - ES. O eugenol (1000 mg/L) foi adquirido em farmácia de manipulação e diluído em álcool etílico comercial $\left(93^{\circ}\right)$ na proporção de 1:10. Esta solução foi armazenada em geladeira. Nos experimentos foi utilizada água tratada colocada previamente para decantação em caixa d'água de polietileno por 48 horas para eliminação do cloro. Foram testadas as seguintes concentrações de eugenol (tratamentos): 40, 60, 80 e $100 \mathrm{mg} / \mathrm{L} \mathrm{em}$ juvenis de oscar com peso e comprimento médio de $4,61 \pm 0,83$ g e $6,36 \pm 0,32 \mathrm{~cm}$. 
Tabela II. Tempo de permanência, em segundos, nos estágios de sedação avaliados na indução anestésica de Astonotus ocelattus com eugenol em diferentes concentraçôes ( $\mathrm{mg} / \mathrm{L}$ ) (Residence time in the sedation stages (in seconds) for Astonotus ocelattus anesthetized using different concentrations ( $\mathrm{mg} / \mathrm{L})$ of eugenol).

\begin{tabular}{|c|c|c|c|c|c|}
\hline \multirow{2}{*}{ Dose } & \multicolumn{5}{|c|}{ Variáveis } \\
\hline & $\mathrm{N}$ & SL & $A L$ & AP & $\mathrm{AC}$ \\
\hline 40 & $18,50(16,81 \%)$ & $35,30(64,87 \%)$ & $99,40(24,48 \%)$ & $159,90(62,83 \%)$ & $571,70(21,16 \%)$ \\
\hline 60 & $21,80(22,57 \%)$ & $31,50(54,51 \%)$ & $71,10(21,76 \%)$ & $77,80(44,07 \%)$ & $259,50(16,47 \%)$ \\
\hline 80 & $27,80(27,99 \%)$ & $17,40(42,63 \%)^{a}$ & $56,90(19,09 \%)$ & $54,30(36,37 \%)$ & $244,00(23,27 \%)$ \\
\hline 100 & $12,20(29,75 \%)$ & $18,80(31,44 \%)^{a}$ & $34,40(25,93 \%)$ & $39,30(16,23 \%)$ & $98,10(77,20 \%)$ \\
\hline
\end{tabular}

Média (Coeficiente de variação). Médias acompanhadas pelas mesmas letras não diferem entre si nas colunas (ANOVA, $\mathrm{p}<0,05)$. N= normal $\left(\hat{Y}=-0,0118 X^{2}+1,5893 X-27,385 ; R^{2}=76,78 \%\right) ; S L=$ Sedação leve $\left(\hat{Y}=0,0032 X^{2}-0,773 X+62,31 ; R^{2}=86,26 \%\right) ; A L=A n e s t e s i a$ leve $\left(\hat{Y}=0,0036 X^{2}-1,5535 X+154,62 ; R^{2}=98,87 \%\right) ; A P=$ Anestesia profunda $\left(\hat{Y}=0,0419 X^{2}-7,7978 X+402,21 ; R^{2}=98,55 \%\right) ; A C=A n e s t e s i a$ cirúrgica $\left(\hat{Y}=0,1039 X^{2}-21,733 X+1253,4 ; R^{2}=92,35 \%\right)$.

Tabela III. Tempo de permanência, em segundos, nos estágios de recuperação avaliados após indução anestésica de Astonottus ocelattus com eugenol em diferentes doses ( $\mathrm{mg} / \mathrm{L}$ ) (Residence time in the recovery stages (in seconds) for Astonotus ocelattus anesthetized using different concentrations (mg/L) of eugenol).

\begin{tabular}{lcrcc}
\hline \multirow{2}{*}{ Dose $(\mathrm{mg} / \mathrm{L})$} & \multicolumn{4}{c}{ Variáveis } \\
\cline { 2 - 5 } & RBO & IM & NE & RF \\
\hline 40 & $23,80(41,13 \%)$ & $131,40(23,51 \%)$ & $75,00(36,56 \%)$ & $177,50(33,03 \%)$ \\
60 & $26,80(20,07 \%)$ & $80,70(38,12 \%)$ & $93,70(39,32 \%)^{\mathrm{a}}$ & $115,00(29,47 \%)^{\mathrm{a}}$ \\
80 & $47,00(27,77 \%)$ & $92,89(39,94 \%)^{\mathrm{a}}$ & $83,89(43,05 \%)^{\mathrm{a}}$ & $112,78(28,52 \%)^{\mathrm{a}}$ \\
100 & $25,00(29,68 \%)$ & $88,90(27,41 \%)^{\mathrm{a}}$ & $94,10(54,30 \%)^{\mathrm{a}}$ & $109,60(51,91 \%)^{\mathrm{a}}$ \\
\hline
\end{tabular}

Média (Coeficiente de variação). Médias acompanhadas pelas mesmas letras não diferem entre si nas colunas (ANOVA, $p<0,05)$. $\mathrm{RBO}=$ Recuperação dos batimentos operculares $\left(\hat{Y}=-0,0156 X^{2}+2,3065 X-46,43 ; R^{2}<70,00 \%\right)$; IM= Início de movimentação ( $\hat{Y}=$

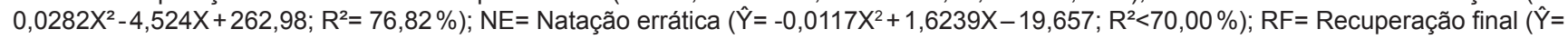
$\left.0,0401 X^{2}-6,6715 X+377,83 ; R^{2}=96,77 \%\right)$.

Os peixes foram obtidos por meio de desova natural em viveiro escavado e levados para crescimento na ala de produção de peixes ornamentais do LNPEO. No laboratório foram alimentados, duas vezes ao dia, com ração extrusada com $32 \%$ de proteína bruta, distribuída a lanço até a saciedade aparente. Antes dos experimentos, os juvenis permaneceram por 24 horas sem alimentação. Os peixes foram medidos com paquímetro analógico e pesados em balança digital com precisão de $0,0001 \mathrm{~g}$; para mensuração dos parâmetros de qualidade de água foram utilizados oxímetro microprocessado modelo DO-5519 e peagâmetro digital modelo PH-0016. Adotou-se o tempo máximo de observação de 30 minutos na recuperação e os peixes que não se recuperassem neste tempo seriam considerados como não recuperados (Vidal et al., 2008).

O experimento foi realizado em delineamento inteiramente casualizado (DIC), com quatro tratamentos e considerou-se cada peixe como uma repetição $(n=10)$. Os peixes foram submetidos à indução anestésica em aquário de vidro contendo um litro de água e após observada completa sedação, caracterizada pela ausência de movimentos e cessação dos batimentos operculares, eram deslocados para recuperação em outro aquário contendo $13 \mathrm{~L}$ de água com aeração constante, realizada por soprador de ar e pedra porosa. Os estágios de sedação e recuperação descritos na tabela I foram acompanhados e avaliados por apenas um observador para todos os tratamentos e repetições. Os tempos, em segundos, de sedação e recuperação foram acompanhados com cronômetro digital.

Os dados referentes ao tempo de permanência em cada estágio de sedação, tempo acumulado para a sedação completa (anestesia cirúrgica) e tempo de recuperação foram analisados por ANOVA $(p<0,05)$ e regressão polinomial utilizando software Sisvar $5.3^{\circledR}$.

\section{RESULTADOS E DISCUSSÃO}

Não houve mortalidade durante o experimento. A temperatura da água no aquário de indução foi de $25,73 \pm 0,34^{\circ} \mathrm{C}$ e no aquário de recuperação foi de $25,66 \pm 0,30^{\circ} \mathrm{C}$, o oxigênio dissolvido apresentou concentração média de $5,45 \pm 0,37 \mathrm{mg} / \mathrm{L}$ e o $\mathrm{pH}$ foi de $7,37 \pm 0,21$. As tabelas II e III mostram os tempos de permanência dos peixes em cada estágio de sedação e recuperação avaliado. A figura 1 mostra a comparação entre os tempos de anestesia completa (A) e recuperação (B) das diferentes concentrações testadas.

Os parâmetros de qualidade de água se mantiveram dentro da faixa de conforto para a maioria das espécies de peixes tropicais (Arana, 2004). Durante a indução à anestesia utilizando as concentrações de 40 e $60 \mathrm{mg}$ / L não ocorreu a hiperatividade inicial descrita por Vidal et al. (2008) em tilápia. Neste trabalho, este comportamento foi observado ao utilizar as doses de 80 e $100 \mathrm{mg} / \mathrm{L}$. Porém, todos os peixes sedados com as doses de 40 e $60 \mathrm{mg} / \mathrm{L}$ apresentaram grande hiperatividade e natação errática na fase final da recuperação, posteriormente voltando à normalidade. Fato não ocorrido para as doses mais altas, 80 e $100 \mathrm{mg} / \mathrm{L}$, que não apresentaram hiperatividade na recuperação.

Observou-se que o aumento da dose diminuiu o tempo de permanência em quase todos os estágios de sedação. Porém, neste trabalho, o aumento da dose não diminuiu o tempo de permanência no estado nor- 

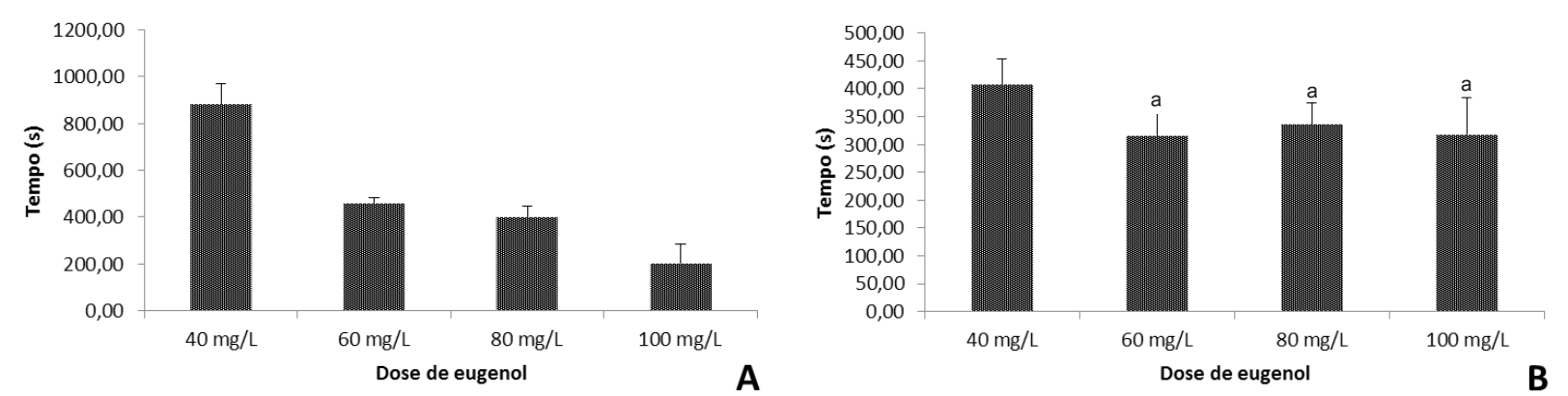

Figura 1. Tempos para anestesia completa (A) e recuperação (B) de Astonotus ocelattus anestesiados com eugenol. Dentro das figuras A e B, colunas acompanhadas pelas mesmas letras não diferem entre si (ANOVA, $\mathrm{p}<0,05)$. Tempos para anestesia completa $(\mathrm{A})\left(\mathrm{Y}=0,1409 \mathrm{X}^{2}-30,268 \mathrm{X}+1845,1 ; \mathrm{R}^{2}=94,98 \%\right)$ e recuperação $(\mathrm{B})$ $\left(\hat{Y}=0,046 X^{2}-7,695 X+634,5 ; R^{2}=80,44 \%\right.$ ) (Time to full anesthesia (A) and recovery (B) for Astonotus ocelattus anesthetized using eugenol. In the Graphics 1 and 2 columns with the same letter are not different from each other (ANOVA, $p<0,05)$. Times to full anesthesia (A) $\left(\hat{Y}=0,1409 X^{2}-30,268 X+1845,1 ; R^{2}=94,98 \%\right)$ and recovery $\left.(B)\left(\hat{Y}=0,046 X^{2}-7,695 X+634,5 ; R^{2}=80,44 \%\right)\right)$.

mal, fato não observado na utilização do eugenol na sedação de algumas outras espécies de peixe (Cunha et al., 2010; Moreira et al., 2010).

Para os outros estágios de sedação, exceto para a permanência no estágio normal, quanto maior foi à dose, menor foi o tempo de permanência nos estágios avaliados, fenômeno constatado também em outros experimentos que utilizaram o eugenol como anestésico para outros peixes (Façanha and Gomes, 2005; Souza et al., 2012; Ribeiro et al., 2013). Este efeito está relacionado ao fato de a solução de eugenol (10\%) ser adicionada na água e quanto maior a dose, maior será a concentração do anestésico na água, o que potencializa o efeito do sedativo acelerando a anestesia completa e reduzindo o tempo de permanência nos estágios de sedação intermediários.

Para os estágios de recuperação (tabela III), observou-se que ocorreu grande variação nos tempos de permanência em cada estágio de recuperação e que apenas no último estágio de recuperação (recuperação final) observou-se que o aumento da dose reduziu o tempo de permanência neste estágio de recuperação, sendo que a dose de $40 \mathrm{mg} / \mathrm{L}$ apresentou o maior tempo de permanência em recuperação final, seguido pelas demais doses testadas, que não diferem entre si.

Elevados coeficientes de variação nos tempos de anestesia e recuperação também foram observados por Diemer et al. (2012) na utilização de eugenol como sedativo para jundiá (Rhamdia sp.). Rotili et al. (2012), em seu trabalho utilizando o eugenol como anestésico para pacu (Piaractus mesopotamicus), observaram que para a sedação ocorre redução no coeficiente de variação dos tempos de indução à medida em que a dose é aumentada, porém isso não se confirma na recuperação.

Para o tempo de anestesia completa (figura 1) observou-se que houve efeito aditivo da dose sobre o tempo de anestesia, de forma que quanto maior a dose, menor o tempo para completa sedação. Este efeito também foi observado quando o eugenol foi utilizado em outras espécies de peixe (Grush et al., 2004; Vidal et al., 2007; Gonçalves et al., 2008; Bittencourt et al., 2013). Como descrito anteriormente, este efeito pode estar relacionado ao aumento da concentração da dose de eugenol, o que pode diminuir o tempo para completa anestesia.

Para a recuperação (figura 1), observou-se que a dose de $40 \mathrm{mg} / \mathrm{L}$ levou ao maior tempo para retomada dos movimentos normais, mas não houve diferença significativa entre os tempos de recuperação dos peixes sedados com as doses de 60, 80 e $100 \mathrm{mg} / \mathrm{L}$. Porém, Bittencourt et al. (2013), utilizando o eugenol na sedação de Carassius auratus observaram que houve aumento do tempo necessário para recuperação à medida que os peixes foram expostos à doses mais elevadas. $\mathrm{O}$ mesmo efeito foi observado por Vidal et al. (2007) na sedação de Leporinus macrocephalus e por Gonçalves et al. (2008) utilizando eugenol em juvenis de pacu.

Os tempos para completa sedação em todos os tratamentos, neste experimento, foram mais altos do que os encontrados por Vidal et al. (2006), que testaram o eugenol em juvenis de pintado (Pseudoplatystoma corrunscans), porém, no presente trabalho observouse menores tempos para a recuperação. Honczaryk and Inoue (2009) testando a eficiência do eugenol na sedação de pirarucu (Arapaima gigas) também observaram menor tempo de sedação e maior tempo de recuperação do que os observados neste experimento com A. ocelattus. Ao comparar a ação do eugenol entre diferentes espécies, deve-se considerar a diferenças metabólicas entre elas (Neiffer and Stamper, 2009). Bittencourt et al. (2013) citam ser possível que a velocidade metabólica da espécie interfira na eficiência dos anestésicos, de forma que espécies com metabolismo mais acelerado e comportamento mais agressivo sejam sedadas mais rapidamente.

O oscar é uma espécie que se comporta apresentando pouca atividade natatória, quando não em atividade de luta ou fuga, o que indica tratar-se de uma espécie que apresenta metabolismo mais lento do que espécies mais ativas como o pintado, o matrinxã (Brycon amazonicus) e o lambari (Asthyanax sp.). A temperatura da água tem grande influência sobre a taxa metabólica dos peixes (Piedras et al., 2004), na sedação das espécies supracitadas com eugenol em água com temperaturas mais baixas do que as do presente trabalho, observa-se que o oscar apresentou maiores tempos para sedação (Inoue et al., 2003; Vidal et al., 2006; Pereira-da-Silva et 
al., 2009). Com base nas equações obtidas para tempo de anestesia total, tempo de permanência em anestesia cirúrgica (margem de segurança) e tempo de recuperação, constatou-se que a dose que causou o menor tempo para anestesia completa foi $107,40 \mathrm{mg} / \mathrm{L}$, sedando os peixes em 219 segundos, esta dose possui margem de segurança de 117 segundos e a recuperação ocorreria em 338 segundos. A dose que causou menor tempo para a recuperação foi $83,64 \mathrm{mg} / \mathrm{L}$, fazendo com que os peixes se recuperassem da sedação em 312 segundos. Esta dose anestesiou os peixes em 299 segundos e permitiu que ficassem em contato com o anestésico por 162 segundos, sem ocorrer a parada dos batimentos operculares. Esta última dose, mesmo com indução mais lenta, apresentou retorno mais rápido e boa margem de segurança. Então, para fins de praticidade, a dose de $80 \mathrm{mg} / \mathrm{L}$ foi a mais indicada para a sedação dos juvenis de oscar.

\section{CONCLUSÃO}

Para a sedação de juvenis de oscar, até a anestesia cirúrgica, com eugenol (10\%) recomenda-se a dose de $80 \mathrm{mg} / \mathrm{L}$ por induzir a sedação e recuperação de forma rápida e oferecer boa margem de segurança após a completa sedação.

\section{BIBLIOGRAFIA}

Anschau, L.; Lazzari, R.; Costa, S.T.; Decarli, J.A.; Uczay, J. e Loebens, L. 2014. Produtos anestésicos para juvenis de carpa húngara (Cyprinus carpio). Rev Bras Saúde Prod Anim, 15: 406-414.

Arana, L.V. 2004. Princípios químicos de qualidade de água em aquicultura: uma revisão para peixes e camarões. $2^{a}$ ed. Editora da UFSC. Florianópolis, SC. Brasil.

Arbalaéz-Rojas, G.A. and Moraes, G. 2009. Interação do exercício de natação sustentada e da densidade de estocagem no desempenho e na composição corporal de juvenis de matrinxã Brycon amazonicus. Ciênc Rural, 39: 201-208.

Ashley, P.J. 2006. Fish welfare: Current issues in aquaculture. Appl Anim Behav Sci, 1: 00-37.

Becker, A.G.; Parodi, T.V.; Heldwein, C.G.; Zeppelfend, C.C.; Heizmann, B.M. and Baldisserotto, B. 2012. Transportation of silver catfish, Rhamdia quelen, in water with eugenol and the essential oil of Lippia alba. Fish Phisiol Biochem, 38: 789-796.

Benovit, S.C.; Gressler, L.T.; Silva, L.L.; Garcia, L.O.; Okamoto, M.H.; Pedron, J.S.; Sampaio, L.A.; Rodrigues, R.V.; Heizmann, B.M. e Baldisserotto, B. 2012. anesthesia and transport of brazilian flounder, Paralichthys orbignyanus, with essential oils of Aloysia gratissima and Ocimum gratissimum. J Aquacult Soc, 43: 896-900.

Bittencourt, F.; Souza, B.E.; Neu, D.H.; Rorato, R.R.; Boscolo, W.R. e Feiden, A. 2013. Eugenol e benzocaína como anestésicos para juvenis de Cyprinus carpio Linnaeus, 1758 (carpa comum). Rev Bras Ciênc Agrárias, 8: 163-167.

Cardoso, R.S.; Lana, A.M.Q.; Teixeira, E.A.; Luz, R.K. e Faria, P.M.C. 2012. Caracterização socioeconômica da aquicultura ornamental na região da zona da mata mineira. Bol Inst Pesca, 38: 89-96.

Costa, P.R.R. 2000. Safrol e eugenol: estudo da reatividade química e uso em síntese de produtos naturais biologicamente ativos e seus derivados. Quím Nova, 23: 357-369.

Cunha, M.A.; Zeppenfeld, C.C.; Garcia, L.O.; Loro, V.L.; Fonseca, M. B.; Emanuelli, T.; Veeck, A.P.L.; Copatti, C.E. and Baldisserotto, B. 2010. Anesthesia of silver catfish with eugenol: time of induction, cortisol response and sensory analysis of fillet. Ciênc Rural, 40: 2107-2114.
Delbon, M.C. and Ranzani Paiva, M.J.T. 2012. Eugenol em juvenis de tilápia-do-nilo: concentrações e administrações sucessivas. Bol Inst Pesca, 38: 43-52.

Diemer, O.; Neu, D.H.; Bittencourt, F.; Signor, A.; Boscolo, W.R. and Feiden, A. 2012. Eugenol as anesthetic for silver catfish (Rhamdia voulezi)with differentweight. Semina: CiêncAgrárias, 33: 1495-1500.

Fabiani, B.M.; Boscolo, W.R.; Feiden, A.; Diemer, O.; Bittencourt, F. and $\mathrm{Neu}$, D.H. 2013. Benzocaine and eugenol as anesthetics for Brycon hilarii. Acta Scientiarum. Anim Sci, 35: 113-117.

Façanha, M.F. e Gomes, L.C. 2005. A eficácia do mentol como anestésico para tambaqui (Colossoma macropomum, Characiformes: Characidae). Acta Amazonica, 35: 71-75.

Gimbo, R.Y.; Saita, M.V.; Gonçalves, A.F.N. e Takahashi, L.S. 2008. Diferentes concentrações de benzocaína na indução anestésica do lambari-do-rabo-amarelo (Astyanax altiparanae). Rev Bras Saúde Prod Anim, 9: 350-357.

Gonçalves, A.F.N.; Santos, E.C.C.; Fernandes, J.B.K. e Takahashi, L.S. 2008. Mentol e eugenol como substitutos da benzocaína na indução anestésica de juvenis de pacu. Acta Sci Anim Sci, 30: 339-344.

Grush, J.; Noakes, D.L.G. and Moccia, R.D. 2004. The efficacy of clove oil as an anesthetic for the Zebrafish, Danio rerio (Hamilton). Zebrafish, 1: 46-53.

Hoseini, S.M.; Rajabiesterabadi, H. and Tarkhani, R. 2015. Anaesthetic efficacy of eugenol in iridescent shark, Pangasius hypophtalmus (Sauvage, 1878) in different size classes. Aquac Res, 46: 405-412.

Holloway, A.C.; Keene, J.L.; Noakes, D.G. and Moccia, R.D. 2004. Effects of clove oil and MS-222 on blood hormone profiles in rainbow trout Oncorhynchus mykiss, Walbaum. Aquac Res, 35: 1025-1030.

Honkzarik, A. and Inove, L.A.K.A. 2009. Anestesia do pirarucu por aspersão direta nas brânquias do eugenol em solução aquosa. Ciênc Rural, 39: 577-579.

Imanpoor, M.R.; Bagheri, T. and Hedayati, S.A.A. 2010. the anesthetic effects of clove essence in persian sturgeon, Acipenser persicus. World J Fish Mar Sci, 2: 29-36.

Inoue, L.A.K.A.; Boinjik, C.L.; Ribeiro, P.T.; Silva, A.M.D. e Affonso, E.G. 2011. Avaliação de respostas metabólicas do tambaqui exposto ao eugenol em banhos anestésicos. Acta Amazonica, 41: 327-332.

Inove, L.A.K.A.; Hackbarth, A. e Moraes, G. 2004. Avaliação dos anestésicos 2-phenoxietanol e benzocaína no manejo do matrinxã Brycon cephalus (Günter, 1869). Biodivers Pampeana, 2: 10-15.

Inove, L.A.K.A.; Santos Neto, C. e Moraes, G. 2003. Clove oil as anaesthetic for juveniles of matrinxã, Brycon cephalus (Gunther, 1869). Ciênc Rural, 33: 943-947.

Magalhães, A.L.B. and Jacobi, C.M. 2013. Invasion risk posed ornamental freshwater fish trade to southeastern Brazilian rivers. Neotropical Ichthiol, 11, 433-441.

Monteiro-Neto, C.; Cunha, F.E.A.; Nottingham, M.C.; Araújo, M.E.; Rosa, I.L. and Barros, G.M.L. 2003. Analysis of the marine ornamental fish trade at Ceará State, northeast Brazil. Biodivers Conserv, 12: 1287-1295.

Moreira, A.G.L.; Teixeira, E.G.; Carreiro, C.R.P. e Moreira, R.L. 2010. Eficácia do eugenol extraído da planta Eugenia aromatica como anestésico para realização de biometrias em adultos de tilápia do Nilo (Oreochromis niloticus). Acta Sci Anim Sci, 32: 419-423.

Neiffer, D.L. and Stamper, M.A. 2009. Fish sedation, anesthesia, analgesia, and euthanasia: considerations, methods, and types of drugs. Inst Lab Anim Res J, 50: 343-360.

Parodi, T.V.; Cunha, M.A.; Heldwein, C.G.; Souza, D.M.; Martins, A.C.; Garcia, L.O.; Wasielesky Júnior, W.; Monserrat, J.M.; Shmidt, D.; Caron, B.O.; Heinzmann, B. and Baldisserotto, B. 2012. The anesthetic efficacy of eugenol and the essential oils of Lippia alba and Aloysia triphylla in post-larvae and sub-adults of Litopenaeus vannamei (Crustacea, Penaeidae). Comp Biochem Phys, 155: 462-468.

Pereira-da-Silva, E.M.; Oliveira, R.H.F.; Ribeiro, M.A.R. e Coppola, M.P. 2009. Efeito anestésico do óleo de cravo em alevinos de lambari. Ciênc Rural, 39: 1851-1856. 
Piedras, S.R.N.; Moraes, P.R.R. e Pouey, J.L.O. F. 2004. Crescimento de juvenis de jundiá (Rhamdia quelen) de acordo com a temperatura da água. Bol Inst Pesca, 30: 177-182.

Ribeiro, F.A.S.; Rodrigues, L.A. e Fernandes, J.B.K. 2007. Desempenho de juvenis de acará-bandeira (Pterophylum scalare) com diferentes níveis de proteína na dieta. Bol Inst Pesca, 33: 195-203.

Ribeiro, P.A.P.; Miranda Filho, K.C.; Melillo Filho, R.; Santos, A.E.H.; Silva, W.S.; Rodrigues, L.A. e Luz, R.K. 2013. Efeito anestésico do eugenol em juvenis de pacamã. Pesqui Agropecu Bras, 48: $1136-1139$.

Rotili, D.A.; Devens, M.A.; Diemer, Lorenz, E.K.; Lazzari, R. e Boscolo, W.R. 2012. Uso de eugenol como anestésico em pacu. Pesqui Agropecu Trop, 42: 288-294.

Simões, S.N.; Paiva, G. e Gomes, L.C. 2010. Óleo de cravo como anestésico em adultos de tilápia-do-nilo. Pesqui Agropecu Bras, 45: 1472-1477.

Souza, R.A.R.; Carvalho, C.V.A.; Nunes, F.F.; Scopel, B.R.; Guarizi, J.D. e Tsuzuki, M.Y. 2012. Efeito comparativo da benzocaína, mentol e eugenol como anestésicos para juvenis de robalo peva. Bol Inst Pesca, 38: 247-255.

Teixeira, E.G.; Moreira, A.G.L.; Moreira, R.L. e Lima, R.F.S. 2011 1. Mentol como anestésico para diferentes classes de tamanho de tilápia-do-nilo. Arch Vet Sci, 16: 75-83.

Trindade, M.E.J. and Queiroz, H.L. 2012. Feeding ecology and morphometry of the digestive tract of Astronotus ocelattus (Cichlidae) em ambientes de várzea environments of the middle Solimões Region, Central Amazon, Brazil. UAKARI, 8: 45-57.

Vidal, L.V.O.; Albinati, R.C.B.; Albinati, A.C.L.; Lira, A.D.; Alameida, T.R. e Santos, G.B. 2008. Eugenol como anestésico para tilápia-do-Nilo. Pesqui Agropecu Bras, 43: 1069-1074.

Vidal, L.V.O.; Albinati, R.C.B.; Albinati, A.C.L. e Mecêdo, G.R. 2006. Utilização do eugenol como anestésico para o manejo de juvenis de pintado (Pseudoplatystoma corruscans). Acta Sci Biol Sci, 28: 275-279.

Vidal, L.V.O.; Furuya, W.M.; Graciano, T.S.; Schamber, C.R.; Santos, L.D. e Soares, C.M. 2007. Concentrações de eugenol para anestesia profunda e toxidade aguda em juvenis de piavuçu (Leporinus macrocephalus). Acta Sci Biol Sci, 29: 357-362.

Wood, C.M.; Iffikar, F.I.; Scott, G.R.; Boeck, G.; Sloman, K.A.; Matey, V.; Domingos, F.X.V.; Duarte, R.M.; Almeida-Val, V.M.F. e Val, A.L. 2009. Regulation of gill transcellular permeability and renal function during acute hypoxia in the Amazonian oscar (Astronotus ocellatus): new angles to the osmorespiratory compromisse. JExp Biol, 212:1949-1964.

Zahl, I.H. and Samuelsen, O. 2012. Anaesthesia of farmed fish: implications for welfare. Fish Physiol Biochem, 38: 201-208.

Zuanon, J.A.S.; Salaro, A.L.; Balbino, E.M.; Saraiva, A.; Quadros, M. e Fontanari, R.L. 2006. Níveis de proteína bruta em dietas para alevinos de acará-bandeira. Rev Bras Zootecn, 35: 1893-1896. 